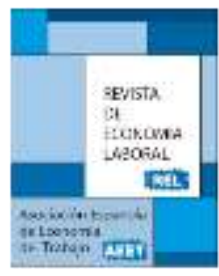

\title{
EL EMPLEO TIC EN ESPAÑA: UN ENFOQUE DE EDAD POR GÉNERO
}

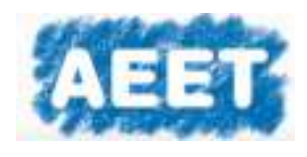

\author{
Diego Dueñas Fernández* y Raquel Llorente Heras** \\ * Universidad de Alcalá e IAES \\ ** Universidad Autónoma de Madrid e IAES
}

Recibido Septiembre 2019; Aceptado Diciembre 2019

\section{Resumen}

En este trabajo se presenta una perspectiva global sobre la situación actual del empleo relacionado con las Tecnologías de la Información y la Comunicación (TIC) en España. Algunos análisis previos predecían un elevado desarrollo de la empleabilidad entre las mujeres dentro las TIC, si bien, tal como demuestra este artículo, la llegada de la crisis ha provocado que no se haya producido dicha tendencia.

Utilizando el análisis de cohortes ficticias, esta investigación analiza el empleo TIC en relación con el género desde una perspectiva generacional. Con ello, se pretende determinar si las TIC han sido una ocupación "refugio" para el empleo femenino así como el destino laboral de las nuevas generaciones de mujeres trabajadoras durante la crisis. Nuestros resultados confirman que el empleo TIC ha ofrecido una ubicación laboral estable y ventajosa para las mujeres. Sin embargo, sigue siendo una ocupación mayoritariamente desarrollada por hombres, en la cual no se ha producido ningún tipo de relevo generacional.

Palabras clave: TIC, cohortes, empleo, jóvenes, género

Clasificación JEL: J13, J16, J44

\begin{abstract}
This paper shows a global perspective of the current employment situation related to Information and Communication Technologies (ICT) in Spain. Some previous analyses predicted a high development of female employability within ICT, although, as this article demonstrates, the economic crisis has caused that this trend has not occurred.

Using fictitious cohorts, this research analyzes ICT employment in relation to gender from a generational perspective. With this goal, the paper intends to determine whether ICT have been an occupation "refuge" for female employment as well as an employment niche for new generations of workers during recent years.

Our findings confirm that ICT employment has resisted the stakes of economic crisis by offering a stable and advantageous work location. However, it remains a mostly male occupation, in which there has been no generational change.
\end{abstract}

Key words: ICT, cohorts, employment, youth, gender

JEL Classification: J13, J16, J44

(C) Revista de Economía Laboral 


\section{Introducción}

En la actualidad, existe un amplio conocimiento sobre las repercusiones de la crisis en términos laborales para hombres y mujeres (Otobe, 2011; Razavi, 2011). Sin embargo, se ha prestado poca atención investigadora al análisis del empleo relativo a las Tecnologías de la Información y la Comunicación (empleo TIC, en adelante), el cual podría jugar un papel clave de cara a la empleabilidad de las mujeres. En el caso de España, existe un alto grado de desconocimiento sobre la evolución reciente de este tipo de empleo, el cual presenta un elevado potencial de integración del colectivo femenino, así como una menor presencia de segregación laboral junto a una discriminación salarial moderada y, a priori, una mayor posibilidad efectiva de conciliación laboral y familiar dadas las mayores oportunidades de flexibilidad que suelen presentarse en este tipo de puestos de trabajo (Díaz-Chao, 2008; Castaño, 2010; Iglesias et al., 2010a; Iglesias et al., 2010b; Iglesias et al., 2010c; Dueñas et al., 2015).

Numerosos trabajos ponen de manifiesto la relación directa entre el empleo relacionado con las TIC y la mejoría que experimenta la posición laboral de las mujeres, en la medida en que este tipo de empleo reduce el uso del esfuerzo físico y lo sustituye por el conocimiento, el trabajo en equipo y la destreza en la capacidad de comunicación (WWWICT, 2004), estimulando de esta forma la demanda de cualificaciones no manuales (Collechia y Papaconstantinou, 1996; Ducatel y Burgleman, 1999; Carnoy, 2002). En el mismo sentido, Chen (2004) demuestra que el desarrollo de infraestructuras relacionadas con las TIC posibilita un mayor nivel de igualdad entre hombres y mujeres, tanto en términos educativos como en términos de empleo.

Pese a ello, las TIC continúan siendo un sector de actividad y/o tipo de ocupación donde la presencia de hombres resulta mayoritaria (Iglesias et al., 2010a). La brecha existente en el empleo por género resulta muy heterogénea según los países y entornos laborales (Castaño et al., 2011; Gil-Juárez et al., 2011; Martínez y Castaño, 2017; Comisión Europea, 2018) pudiéndose afirmar que se ha mantenido a lo largo del período de crisis. Asimismo, dentro del empleo TIC persiste un sistema dual donde los hombres tienden a colocarse en los puestos de trabajo más técnicos y cualificados mientras que las mujeres se ocupan con mayor frecuencia en puestos de trabajo menos especializados, sin considerar en dicho reparto las habilidades o cualificaciones específicas de cada grupo. Esta brecha ocupacional existente dentro de la brecha de empleabilidad o acceso laboral a este tipo de empleos se conoce como Doble Brecha 
Dueñas y Llorente / Revista de Economía Laboral 16(2) (2019), 38-68

Digital analizada profundamente en el caso español por Castaño et al. (2008).

El presente artículo cuenta con varios objetivos claves. En primer lugar, se pretende ofrecer una visión actual sobre cuál ha sido la evolución reciente del empleo TIC desde una doble perspectiva. Por un lado, desde un enfoque longitudinal se analizará la capacidad de incorporación a este tipo de empleo que tienen las mujeres y los hombres. Con ello, se comprobará si las ocupaciones TIC han sido un "refugio" para el empleo durante la crisis. En segundo lugar, pretendemos analizar si se ha producido un acceso de las nuevas generaciones al empleo TIC, es decir, si las mujeres jóvenes se han incorporado a este tipo de puestos de trabajo. Ello permitirá conocer si las nuevas generaciones de mujeres, con respecto a las anteriores, han encontrado en el empleo TIC un nicho laboral en el cual puedan desarrollar las capacidades obtenidas en la etapa de formación académica (Chen, 2004). Es un hecho conocido que la formación se ha incrementado sustancialmente entre las mujeres en los últimos años. Según la Encuesta de Población Activa (de aquí en adelante $\mathrm{EPA}$ ) el 5,5 por ciento de las mujeres mayores de 16 años poseen estudios superiores, mientras que la misma cifra para el caso de los hombres es del 5 por ciento․ Para desarrollar ambas cuestiones, se planteará como herramienta metodológica la utilización del análisis de cohortes ficticias quinquenales (Toharia et al., 1997; Garrido y Chuliá, 2005; Malo y Cueto, 2014).

Por otra parte, se analizará el grado de estabilidad laboral que proporcionan los empleos TIC, considerando como tal el número de años de experiencia que acumulan los trabajadores en la misma empresa. Este análisis también se estructurará a través del análisis de cohortes ficticias pero en relación a la experiencia laboral, tal como se explicará posteriormente en el apartado metodológico.

Para desarrollar los objetivos previos, el trabajo se estructura de la siguiente manera. A continuación, se desarrolla una breve recopilación de bibliografía sobre el tema de análisis intentando centrar la atención en la novedad de las investigaciones. Posteriormente, se aclaran algunas cuestiones metodológicas desarrolladas en el artículo. En el siguiente apartado se presenta una panorámica general sobre la evolución del empleo TIC en España, prestando especial atención a la perspectiva de género. Tras este apartado se lleva a cabo el análisis longitudinal de cohortes ficticias que permite contrastar la empleabilidad mostrada por este tipo de empleo para las nuevas generaciones y la estabilidad del

\footnotetext{
${ }^{1}$ Datos relativos al segundo trimestre de 2019.
} 
empleo TIC a lo largo de los últimos nueve años. Asimismo, se analizará cuál es la presencia en el empleo TIC de las distintas cohortes de edad, teniendo en cuenta las posibles diferencias por género. Complementariamente, se desarrolla un análisis econométrico sobre la probabilidad de encontrarse ocupado u ocupada en el entorno de las TIC. Finalmente, se evaluará la estabilidad en el empleo TIC utilizando como indicador los meses de permanencia en la misma empresa. En el último apartado se recopilan las principales conclusiones del estudio realizado.

\section{Análisis reciente de las TIC en el ámbito académico}

En términos generales, la difusión de las nuevas TIC en España comenzó a finales de los años noventa. A partir de este momento surgieron numerosos e interesantes estudios en relación a sus mayores posibilidades de empleabilidad, mejores condiciones laborales y menor segregación femenina (Díaz-Chao, 2008; Castaño, 2010; Iglesias et al., 2010a; Iglesias et al., 2010b; Iglesias et al., 2010c; Dueñas et al., 2015). El estudio de referencia durante este periodo fue el informe titulado "WWW-ICT" (2004) resultado del proyecto europeo "Information Society Technologies, 5th Framework Programme"2 que analizaba el mercado de trabajo de siete países europeos y establecía un primer conocimiento sobre la situación laboral de las mujeres en las TIC (desafortunadamente España no estaba incluida). En todas estas investigaciones se preveía un amplio desarrollo del empleo TIC en el futuro y, con ello, una elevada incorporación de las mujeres a este tipo de empleo. Además, las TIC parecían ser una ubicación laboral con un elevado potencial para la igualdad de género y que podrían ser un ejemplo a seguir para evitar la segregación laboral. Sin embargo, la llegada de la crisis y el aumento generalizado del desempleo provocó que no se cumplieran dichas expectativas, tal como se demostrará posteriormente.

Con la llegada de la crisis, las TIC permanecen siendo una ubicación laboral con un mayor porcentaje de empleo entre los hombres (Castaño et al., 2011; Gil-Juárez et al., 2011; Martínez y Castaño, 2017; Comisión Europea, 2018). La causa de ello no se puede atribuir a un solo factor; el contexto social y político, la cultura general, la regulación laboral, los roles de género preestablecidos, las desiguales preferencias personales, las distintas habilidades de los trabajadores, su formación y educación, etc. son algunas de las infinitas causas que se establecen como

\footnotetext{
2 Para un mayor conocimiento se recomienda consultar: http://www.ftu-namur.org/www-ict/ (consultado el 24/11/2019).
} 
explicación de la desigual colocación laboral por género. Asimismo, la Segunda Brecha Digital predicha por Castaño et al. (2008) se mantiene durante la crisis, de modo que, dentro de las TIC persiste un sistema dual. Determinados estudios (Arroyo, 2007; Arroyo y Valenduc, 2016) demuestran que las mujeres ocupan con mayor frecuencia puestos de trabajo donde se requieren cualificaciones sociales, comunicativas y de atención personal denominadas en la literatura especializada "hybrid skills" cuya traducción literal puede ser cualificaciones híbridas, en contraposición a las habilidades técnicas, de manera que incluso dentro de las TIC el sistema dual de empleo reproduce los estereotipos de colocación por género tradicionales. Todo ello en un entorno donde existe una importante polarización de los puestos de trabajo dentro de las TIC que acentúa la brecha existente entre los trabajadores cualificados y técnicos frente al resto (OCDE, 2017).

En relación a las nuevas cohortes de edad, Castaño (2009) muestra que existe una amplia difusión en el uso de las TIC entre los hombres y mujeres jóvenes. Sin embargo, la igualdad en la tenencia de las habilidades digitales por género no conlleva una igualdad en el empleo. Asimismo, la elección de estudios técnicos relacionados con las TIC también se encuentra segregada hacia los hombres (Fernández et al., 2008; Sanz, 2008). La formación técnica desigual por género es otra de las posibles causas o explicaciones de la segregación laboral femenina en las TIC. Por consiguiente, aunque existe un escenario favorable hacia la incorporación de las mujeres a las TIC, dicha pauta no parece producirse.

Durante el periodo de crisis, de acuerdo con el estudio de la Comisión Europea (2018) los trabajadores de las TIC de edades más avanzadas o "mayores", aquellos con más de 44 años de edad, han tendido a transitar hacia la jubilación con gran intensidad. Ello se ha debido principalmente a los incentivos dados por las empresas para acceder a la jubilación anticipada. Además, dicha pauta ha resultado algo más fuerte entre las mujeres.

La brecha salarial por género se mantiene dentro de los puestos de trabajo TIC y se agudiza en contra de la mujer en los sectores/ocupaciones más cualificados o intensivos debido a las condiciones laborales más exigentes existentes en dichas ubicaciones laborales (Iglesias et al., 2010b; Segovia-Pérez et al., 2019). A pesar de ello, debe destacarse que las TIC ofrecen puestos de trabajo más estables y duraderos con una mayor remuneración frente al resto de ubicaciones laborales (Iglesias et al., 2010b). El trabajo de la Comisión Europea (2018) demuestra que dentro de las TIC existe una elevada dificultad 
para conciliar vida laboral y familiar debido a la fuerte dedicación que exige este tipo de empleos. Las posibilidades de flexibilización horaria, deslocalización o trabajo desde el hogar que a priori presentan las TIC no termina de favorecer la incorporación femenina (Iglesias et al., 2010c). Adicionalmente se observa como en la carrera laboral femenina dentro de las TIC existe un "fenómeno de abandono"3 entre los 30 y 40 años de edad, momento que coincide con la tenencia de hijos (Comisión Europea, 2018).

Finalmente, destacar que los esfuerzos por aumentar la presencia de las mujeres en los entornos TIC se han incrementado considerablemente en los últimos años (Caridad y Ayuso, 2011). Propuestas de visualización de las mujeres empleadas en los ámbitos TIC, el establecimiento de ejemplos a seguir, fomentar la formación no excluyente en ámbitos técnicos, hacer atractivo el uso de la tecnología, facilitar el acceso a las TIC en ámbitos rurales, etc. han sido algunas de las acciones y propuestas realizadas para reducir la brecha digital por género (Gil-Juárez et al, 2011; Cabero y Ruiz, 2018).

\section{Cuestiones metodológicas}

La base de datos que se utiliza en este trabajo es la Encuesta de Población Activa (EPA) suministrada de forma trimestral por el Instituto Nacional de Estadística (INE). Se trata de la encuesta de referencia a la hora de estudiar los principales aspectos referidos al mercado laboral y sirve de base para el cálculo de las tasas laborales básicas. El periodo analizado comprende los segundos trimestres de los años 2011 a 2019, alcanzando los datos más recientes en el momento de elaboración de esta investigación. La elección del periodo de análisis viene motivada por dos razones: en primer lugar, a partir del año 2011 entró en vigor la nueva Clasificación Nacional de Ocupaciones (CNO-2011) lo cual supone un importante cambio metodológico. Solo a partir de este año se consigue una serie homogénea en el análisis ocupacional. En segundo lugar, al asumir este periodo de estudio, el presente trabajo amplía y continúa la investigación realizada en Dueñas et al. (2015) permitiendo comparar los resultados obtenidos previamente en un entorno de crisis laboral. La elección de los segundos trimestres se justifica porque el ciclo económico

\footnotetext{
3 Traducido de la expresión inglesa "drop-out phenomenon" concepto sobre el cual existe toda una teoría al respecto en relación con las diferencias por género y la voluntariedad implícita.
} 
trimestral se asemeja a la media anual evitando realizar un mayor número de cálculos y estimaciones.

En nuestro caso particular, para el análisis de las TIC, la EPA incluye un nivel de desagregación a tres dígitos tanto en sectores de actividad como en la escala ocupacional. Este nivel de desagregación es necesario para analizar todo lo concerniente al empleo en las TIC. La Organización para la Cooperación y el Desarrollo Económicos (OCDE) define este tipo de empleo como la parte del empleo total relacionado especialmente con las Tecnologías de la Información y la Comunicación, para lo cual utiliza una doble aproximación (OCDE, 2004): por una parte, el empleo TIC se correspondería con aquel tipo de empleo que se encuentra ubicado en los sectores productores de bienes y servicios TIC; por otra, el empleo TIC se refiere a aquellas ocupaciones que muestran una especial relación con las TIC. Dicho de otra forma, la primera perspectiva se refiere a cualquier empleo que esté situado en un sector de actividad relacionado con las TIC, independientemente de que dicho trabajo tenga algún tipo de relación con la tecnología. La segunda definición tiene en cuenta la utilización de las TIC directamente en el desarrollo del puesto de trabajo, independientemente de que dicho empleo esté situado en una actividad relacionada con las TIC.

A partir de esta doble perspectiva, este trabajo considera como más conveniente la segunda aproximación ya que se entiende que la ocupación tiene una mayor aproximación al uso de las tecnologías TIC (Dueñas et al., 2015). Por tanto, siguiendo la definición más actual y difundida en el mundo académico del empleo TIC (OCDE, 2014), la tabla I presenta las ocupaciones consideradas dentro de este tipo de empleo en el mercado de trabajo español ${ }^{4}$.

\footnotetext{
${ }^{4}$ Los autores han traducido la clasificación internacional ISCO-08 utilizada por la OCDE a la clasificación nacional CNO-11 a través de las correspondencias establecidas por el INE.
} 
Tabla I: Definición de las ocupaciones TIC. Clasificación Nacional de Ocupaciones, 2011 (CNO-11).

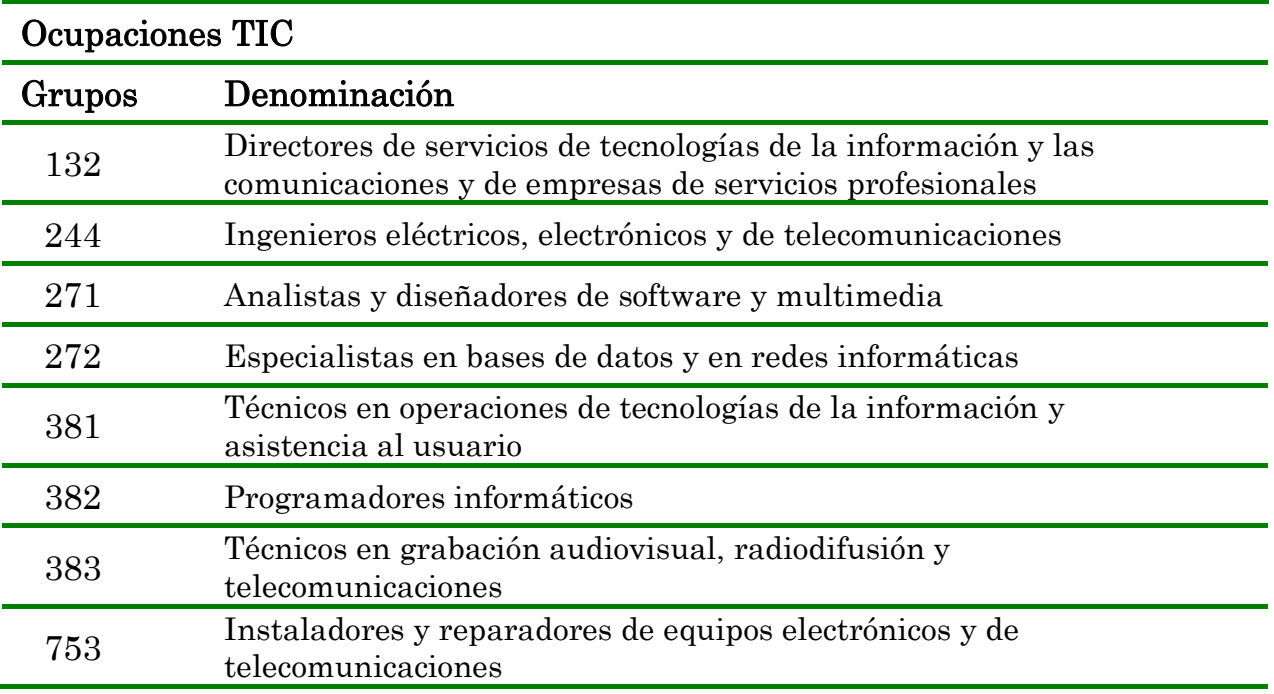

Las ocupaciones TIC se comparan con el resto de ocupaciones. Esta metodología es implementada por la OCDE (2004 y 2014) y replicada en el caso español (Iglesias et al., 2010a). Es la comparación más directa y sencilla ante la falta de una comparación con ocupaciones que podamos identificar como similares, pues las cualificaciones y formación requeridas para ejercer las ocupaciones TIC (ubicaciones laborales aún en desarrollo) no se corresponden con las de otras ocupaciones.

En la investigación para desarrollar nuestros análisis se estimarán dos índices sencillos que se explican a continuación:

- $\quad$ El Índice de Karmel y MacLachlan (Karmel y MacLachlan, 1988) que mide la segregación laboral, es decir, la distribución en el empleo de hombres y mujeres. Se trata de un índice ampliamente conocido que se calcula como:

$K M=\frac{1}{T} \sum_{i=1}^{N}\left|\frac{F}{T} m_{i}-\frac{M}{T} f_{i}\right|$

Donde, "f. es el porcentaje de mujeres en la ocupación i y " $\mathrm{m}_{\mathrm{i}}$ " es la misma ratio para los hombres; $\mathrm{F}$ es el número total de mujeres empleadas, $\mathrm{M}$ es igual para el caso masculino y $\mathrm{T}$ es el número total de trabajadores. Este índice mide el porcentaje del empleo 
total que debería cambiar de ocupación, con reposición, para alcanzar un grado de segregación por sexo nulo o inexistente.

Asimismo, este índice de segregación laboral es adecuado cuando se comparan grupos ocupacionales distintos y ambos grupos tienen tamaños distintos, ya que tiene en cuenta el tamaño relativo del empleo masculino y femenino, a diferencia de otros índices para medir la segregación laboral usualmente utilizados, como por ejemplo el Índice de Duncan y Duncan (Duncan y Duncan, 1955). Principalmente por este motivo, se considera por algunos autores uno de los indicadores más adecuados para medir la segregación laboral por género a lo largo de un periodo de tiempo (Rendón y Salas, 2000).

- En segundo lugar, se estimará el Coeficiente de Asimetría de Fisher que analiza la distribución de una variable en función de los valores observados en torno a la media. También se trata de una medida muy difundida en Estadística que calcula:

$\gamma_{1}=\frac{\mu_{3}}{\sigma^{3}}$

Donde $\mu_{3}$ es el tercer momento en torno a la media y $\sigma$ es la desviación estándar, de modo que si:

- $\gamma_{1}>0$ la distribución es asimétrica a la derecha, es decir, los valores de la derecha cuentan con una mayor representación o presencia.

- $\gamma_{1}<0$ la distribución es asimétrica a la izquierda, es decir, los valores de la izquierda cuentan con una mayor representación o presencia.

- $\gamma_{1}=0$ la distribución es simétrica. Existe un reparto de representación entre todos los valores que puede tomar una variable.

Por consiguiente, determina la desigual distribución de una variable respecto a su media. En nuestro caso, se utilizará para detectar asimetrías en la distribución por edad de la población joven en las ocupaciones TIC y determinar cómo es la inserción laboral. Asimismo, este índice se calculará para hombres y mujeres.

El desarrollo del trabajo también supondrá la realización de un análisis de cohortes ficticias. Esta metodología consiste en enfocar el análisis y la investigación en el seguimiento de un grupo construido a partir de su edad. En nuestro caso, estas cohortes se aúnan por quinquenios. Se denomina ficticia porque no se analizan a las mismas 
personas a lo largo del tiempo, sino que se observa cuál es la situación de una misma generación o cohorte, es decir, se observa la evolución del grupo representativo de una cohorte a lo largo del tiempo.

Por último, en la parte final del trabajo se desarrolla un modelo econométrico sobre la probabilidad de estar empleado en una ocupación TIC frente a estar empleado en el resto de ocupaciones no TIC controlando por la desigual participación laboral. El modelo seleccionado se trata de un "Probit' con sesgo de selección según Heckman (Heckman, 1979). Tener en cuenta el sesgo de selección resulta fundamental en la economía española donde la participación laboral difiere entre géneros. La elección de un modelo "Probit" radica en la posibilidad de calcular los efectos marginales que permiten una comparación de los resultados más sencilla. Las variables independientes consideradas recogen las principales características personales y laborales de los trabajadores (Ser mujer, tramos de edad en quinquenios, estar casado, ser padre o madre, tener estudios secundarios, tener estudios superiores, trabajar a jornada completa, trabajar bajo un contrato indefinido y dummies relativas a las Comunidades Autónomas). Entre las variables a contrastar destaca la variable dicotómica "tener un contrato cuya duración del contrato sea mayor a 2 años", dado que puede establecer si existe una cierta rotación o estabilidad en el empleo TIC.

\section{Evolución reciente y posición del empleo TIC en España}

El gráfico 1 muestra cuál ha sido la evolución del empleo TIC en España durante los últimos años. Como información complementaria, en el eje de abscisas se incluye el porcentaje de empleo TIC con respecto al empleo total para los segundos trimestres del periodo comprendido entre los años 2011 y 2019, el cual ha aumentado desde el 3,3 por ciento hasta el 3,7 por ciento.

En este gráfico se puede observar que las ocupaciones TIC presentan una fuerte resiliencia en los periodos de crisis, ya que entre los años 2011 y 2013 este tipo de empleo perdió únicamente el 4,82 por ciento de ocupados. Por otra parte, se puede establecer que este tipo de empleo presenta un fuerte dinamismo y capacidad generadora de puestos de trabajo en periodos de relativa recuperación económica. Entre 2013 y 2019, el número de ocupados TIC ha aumentado en un 29,18 por ciento, muy por encima del incremento porcentual del resto de ocupaciones. 
Gráfico 1: Evolución del número de ocupados en el empleo TIC, y peso porcentual de dicho empleo con respecto al empleo total. Segundos trimestres, 2011 - 2019. (Fuente: EPA, INE).

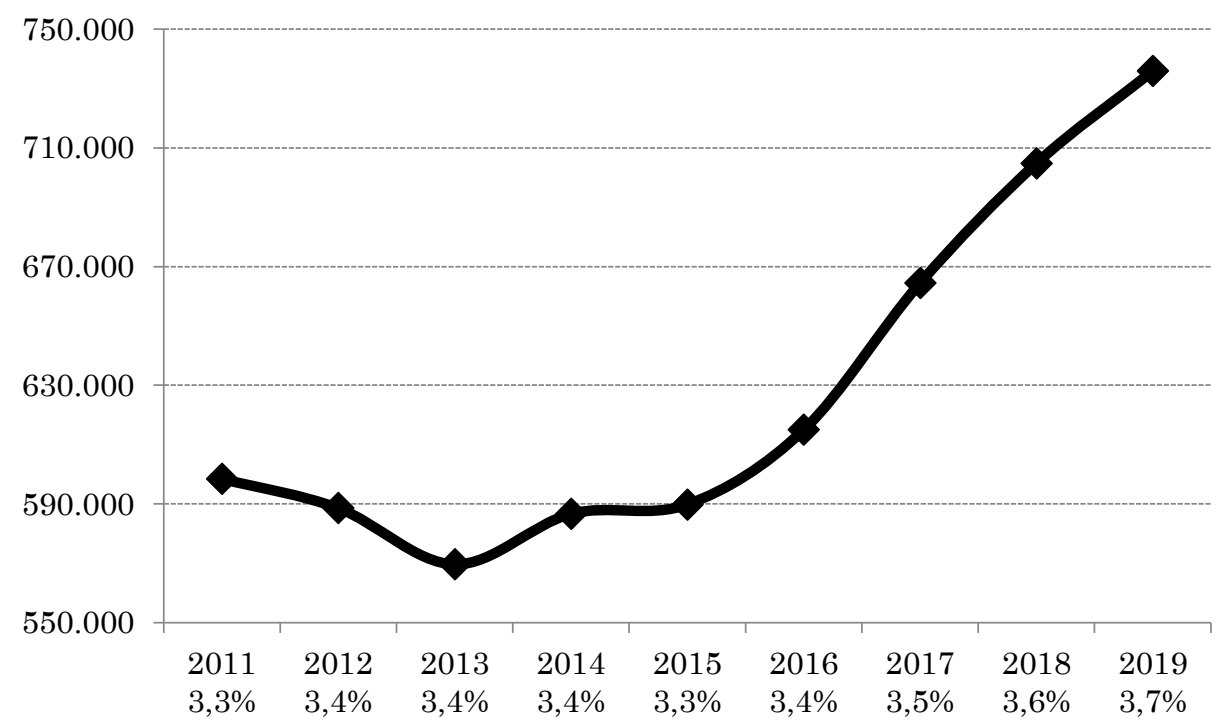

En el gráfico 2 se analiza cuáles son las características básicas del empleo TIC estimando su distribución según el tipo de contrato (sea o no indefinido) y de jornada laboral (sea o no completa). De su observación se desprende que el empleo de las ocupaciones TIC tiene un mayor grado de estabilidad laboral que el resto de ocupaciones. A lo largo de todo el periodo considerado es posible concluir que las ocupaciones TIC tienen un mayor porcentaje de trabajadores con contrato indefinido (11,28 puntos porcentuales más, en promedio) y presentan con mayor frecuencia el desarrollo de jornadas laborales a tiempo completo (11,10 puntos porcentuales más, en promedio). 
Gráfico 2: Incidencia de los contratos indefinidos y la jornada completa en el empleo TIC y no TIC. Segundos trimestres, 2011, 2015 y 2019. (Fuente: EPA, INE).

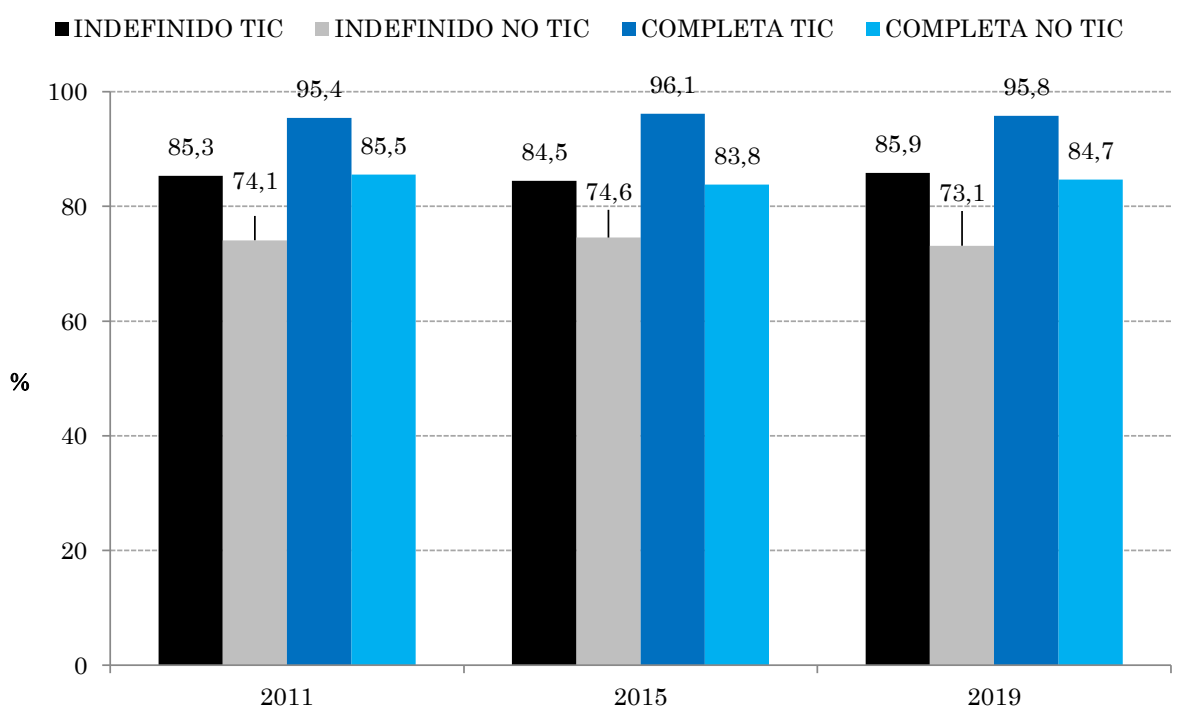

Gráfico 3: Evolución del número de ocupados por género en el empleo TIC. Segundos trimestres, 2011 - 2019. (Fuente: EPA, INE).

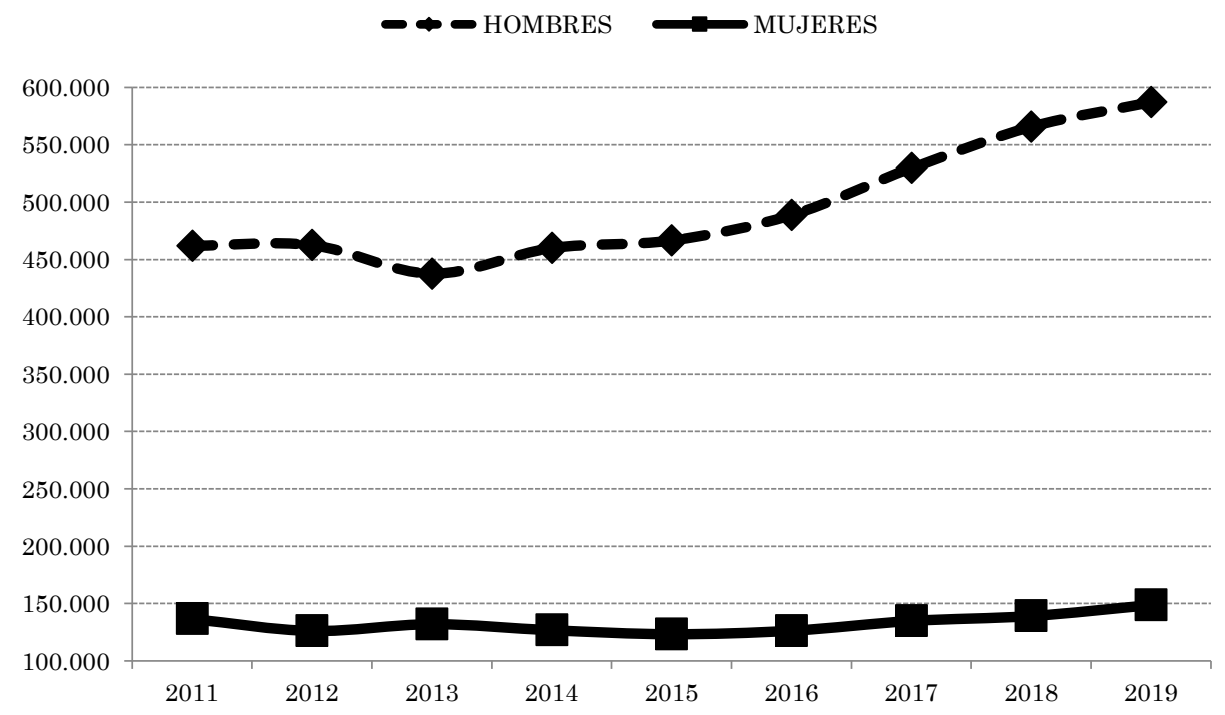


Centrando el foco de atención en el objetivo principal de este trabajo, el gráfico 3 muestra la evolución del número de ocupados que ha experimentado el empleo TIC, de forma separada para hombres y para mujeres en el mercado de trabajo español. El 80 por ciento de los empleos relativos a las ocupaciones TIC han sido ocupados durante todo el periodo por hombres siendo tan solo el 20 por ciento restante ocupados por mujeres $^{5}$. A lo largo de los últimos nueve años, el número de hombres empleados dentro de las TIC se ha visto incrementado en un 27,10 por ciento mientras que el número de las mujeres empleadas en las ocupaciones TIC solamente ha crecido un 8,92 por ciento.

Gráfico 4: Índice de segregación ocupacional de Karmel y MacLachlan. Segundos trimestres 2011 - 2019. (Fuente: EPA, INE).

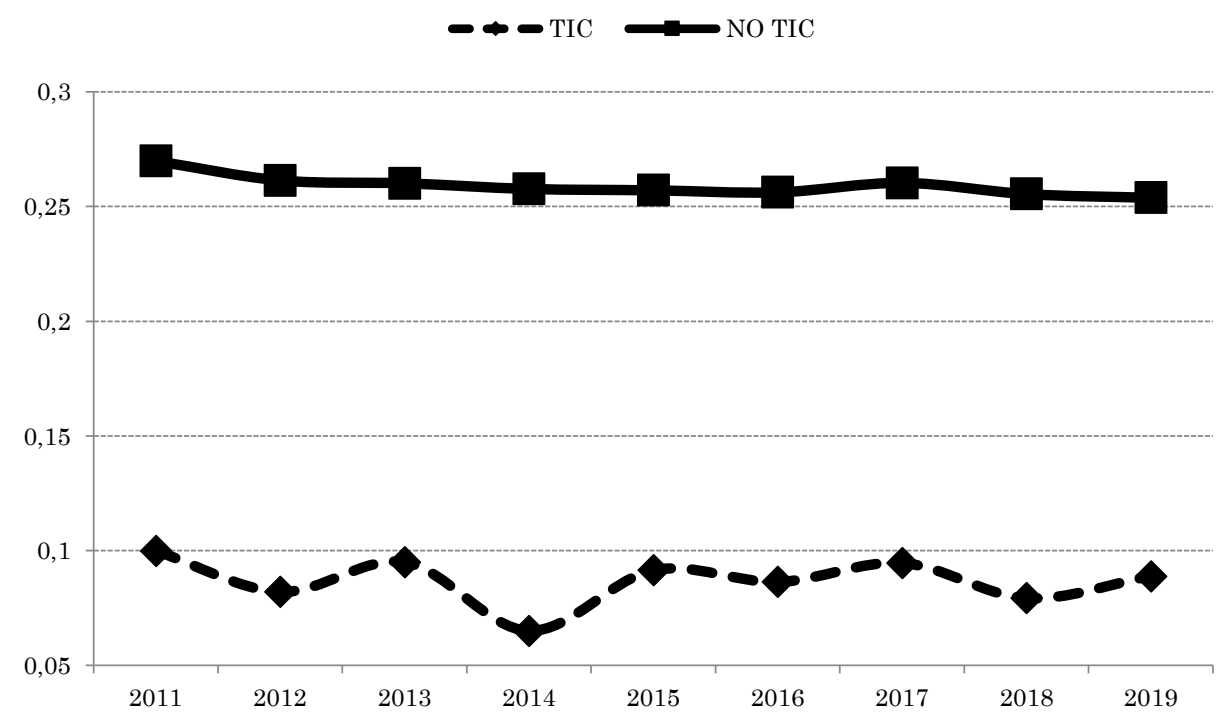

\footnotetext{
5 Estos porcentajes guardan una estrecha relación con la distribución por género de los matriculados y los egresados en los grados universitarios vinculados a las ingenierías y a la arquitectura. Utilizando datos del Ministerio de Ciencia, Innovación y Universidades, en los últimos años el 25 por ciento de las matrículas y el 28 por ciento de los egresados eran mujeres. En el mismo sentido, Fernández et al. (2008) y Sanz (2008) demuestran que la elección de estudios técnicos relacionados con las TIC se encuentra segregada hacia los hombres.
} 
Pese al incremento del empleo entre los hombres, el gráfico 4 pone de manifiesto que las ocupaciones TIC son una ubicación laboral con un nivel muy reducido de segregación laboral. De acuerdo con el cálculo desarrollado del Índice de Karmel y MacLachlan la distribución por género del empleo dentro de las ocupaciones TIC es más igualitaria que en otros escenarios laborales. El nivel de segregación para las ocupaciones TIC asciende a 0,0869 mientras que para las ocupaciones no TIC es de 0,2591. Es decir, la segregación ocupacional del empleo TIC supone un tercio de la segregación del resto de ocupaciones. Este promedio indica que en el empleo TIC solamente un 8,69 por ciento del empleo total debería cambiar de ocupación, con reposición, para alcanzar un grado de segregación nulo ${ }^{6}$. Del mismo modo, el porcentaje de rotación laboral en el resto de ocupaciones - las ocupaciones no TIC - debería ser un 25,91 por ciento.

Los gráficos anteriores muestran una primera perspectiva de lo que significa el empleo TIC en el mercado de trabajo español. En este sentido, ha sido un tipo de empleo en constante crecimiento desde el año 2013, incrementando su peso porcentual dentro del empleo total. Además, se trata de un tipo de empleo que atesora cierta estabilidad laboral dado que presenta un mayor porcentaje de trabajadores indefinidos y con jornada a tiempo completo. Por último, en cuestiones de género es un empleo con un porcentaje elevado de hombres (alrededor de un 80 por ciento), si bien las ocupaciones TIC presentan un nivel de segregación laboral muy inferior al que presentan el resto de ocupaciones ${ }^{7}$. Es decir, el reparto del empleo por género dentro de las ocupaciones TIC resulta menos desigual que a nivel general o para el resto del empleo.

\section{Análisis del empleo TIC: edad, cohorte y periodo}

A través de esta metodología se pretende validar dos de los objetivos del trabajo. Por un lado, si las ocupaciones TIC son un destino laboral para los demandantes de empleo más jóvenes, y, por otro, si las ocupaciones TIC contribuyen a la sostenibilidad y estabilidad laboral

\footnotetext{
${ }^{6}$ Es decir, para que exista la misma proporción de mujeres y hombres en cada ocupación que la que existe a escala agregada en todo el empleo TIC, manteniendo constante al mismo tiempo la estructura ocupacional y los porcentajes de participación de cada sexo en el empleo total.

${ }^{7}$ Lo cual a priori puede ser indicativo de una menor discriminación salarial en contra de las mujeres, dada la estrecha relación que existe entre ambas cuestiones (Palacio y Simón, 2006).
} 
para las generaciones maduras. Como primera aproximación a este análisis, el gráfico 5 muestra la evolución que ha experimentado la edad media de los empleados en las ocupaciones TIC entre 2011 y 2019 . A la vista del gráfico 5, se aprecia un ligero aumento en la edad media de los empleados TIC. En el periodo 2011 a 2019 la edad media de los trabajadores TIC ha aumentado en algo más de 2 años, pasando de 38,4 a 40,7 años de edad, de manera que existe un cierto "envejecimiento" de la mano de obra colocada en estas ubicaciones laborales. A priori, todo parece indicar que los trabajadores más jóvenes han perdido peso relativo o presencia en el empleo TIC en la última década.

Gráfico 5: Evolución de la edad de los trabajadores ocupados en el empleo TIC. Segundos trimestres 2011 - 2019. (Fuente: EPA, INE).

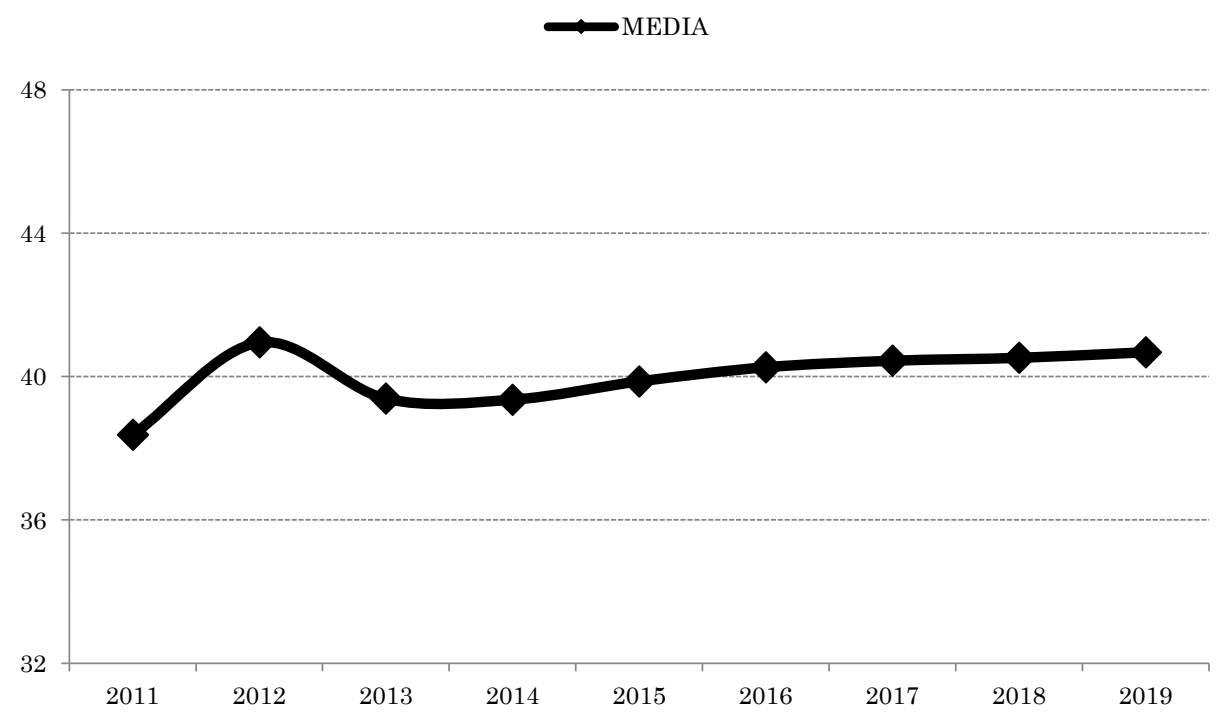

5.1. La empleabilidad de los jóvenes en las ocupaciones TIC

En este apartado se pretende contrastar si la empleabilidad de las generaciones más jóvenes se ha incrementado dentro de las ocupaciones TIC durante los últimos años. Para ello, se desarrolla un análisis de cohortes ficticias. Se trata de establecer ciertos tramos de edad de la población objeto de estudio, en nuestro caso, los trabajadores TIC en quinquenios de edad ${ }^{8}$. Posteriormente, se analiza cuál es la distribución

8 Concretamente, se analizan nueve tramos de edad. El primero se corresponde al rango 21-24 años y el último al tramo de edad que va desde los 60 años en adelante. Se ha 
porcentual que presentan dichos tramos en distintos momentos del tiempo estableciendo cuál es el cambio de su estructura entre 2011 y 2019.

Ello nos permite observar si el paso del tiempo provoca un "envejecimiento" de las ocupaciones TIC debido a que pesan más las generaciones más maduras o de mayor edad; este hecho se aprecia en que la distribución por edad se vuelve más asimétrica y se ve sesgada hacia la derecha (siempre que se coloquen los trabajadores de mayor edad en la derecha de la distribución). O, por el contrario, las ocupaciones TIC se "rejuvenecen", en favor de las generaciones más jóvenes pudiendo observar que la distribución de cohortes por edad se vuelve más asimétrica hacia la izquierda (de acuerdo con una ordenación estándar de cohortes jóvenes a maduras, de izquierda a derecha respectivamente).

En el gráfico 6 se muestra la evolución de la distribución obtenida a lo largo de los años 2011, 2015 y 2019 para el total del empleo TIC y diferenciando por género. Se puede observar que existe desplazamiento hacia la derecha de la distribución. Ello supone que las cohortes de menor edad han ido perdiendo peso porcentual con el paso de los años en favor de las cohortes más longevas. Por ejemplo, los trabajadores TIC entre 25 y 29 años suponían un 14,95 por ciento en el año 2011. Mientras que en el año 2019 representan el 10,20 por ciento del total del empleo TIC. Por el contrario, la cohorte entre 45 y 49 años ha pasado de representar un 12,36 por ciento en 2011 a un 15,45 por ciento en 2019 .

Para el caso de los hombres se alcanza una conclusión similar a la obtenida previamente. La distribución del empleo TIC por cohortes de edad ha experimentado un desplazamiento hacia la derecha en los últimos nueve años. Se produce una pérdida de representación de las cohortes de hombres más jóvenes a favor de las cohortes de hombres mayores. La cohorte de hombres entre 30 y 34 años pasa de un 21,24 por ciento en 2011 a un 14,74 por ciento en 2019 .

suprimido el tramo de edad 16-20 por la falta de representatividad muestral entre las mujeres. 
Gráfico 6: Distribución porcentual por cohortes de edad en el empleo TIC para todos los ocupados, hombres y mujeres. Segundos trimestres 2011, 2015 y 2019. (Fuente: EPA, INE).
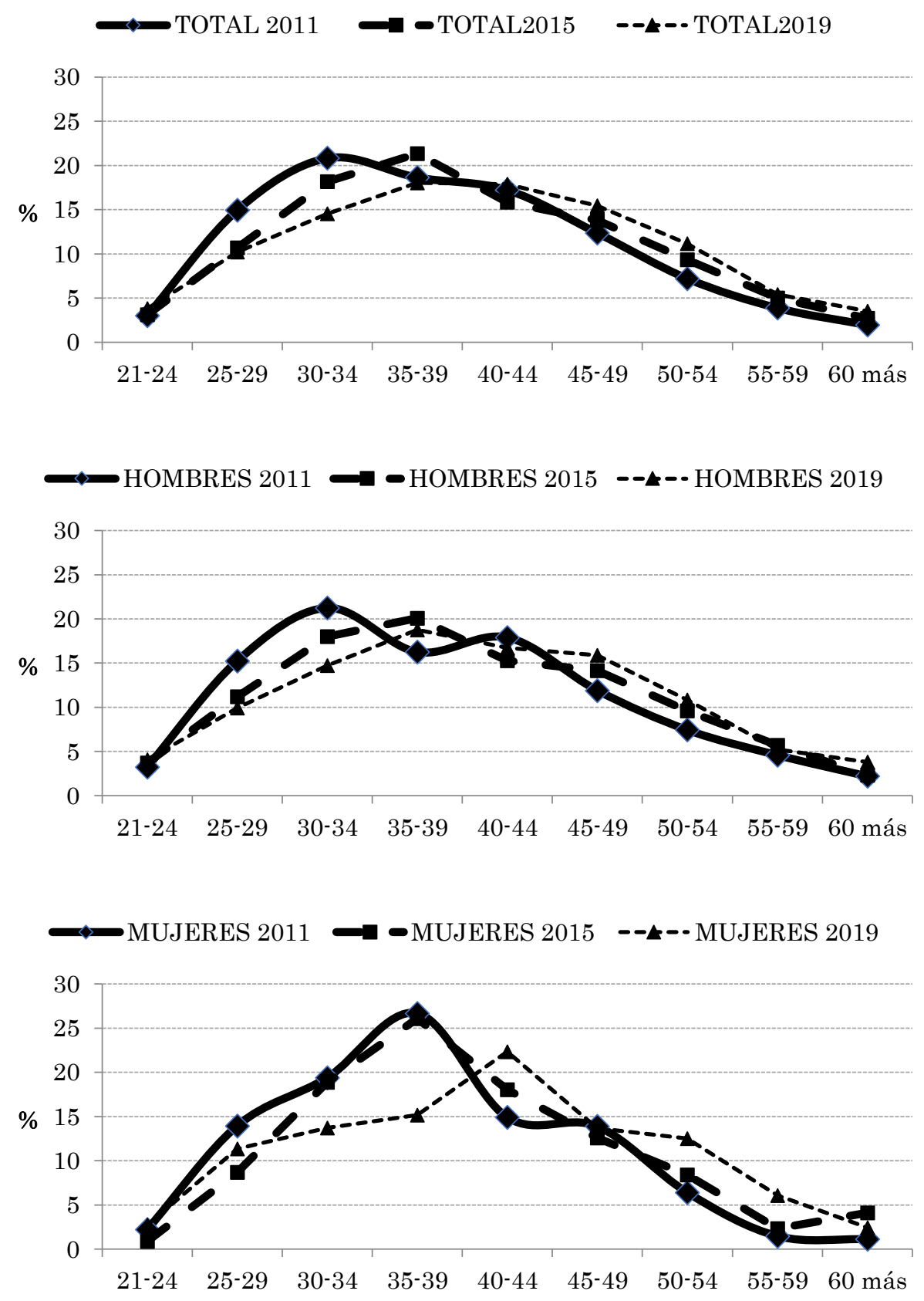
En el gráfico 6 la dinámica para las mujeres no dista mucho de la que han seguido los hombres. Se observa un desplazamiento hacia la derecha de la distribución obtenida por cohortes de edad estableciendo un cierto "envejecimiento" del empleo TIC o la falta de representación de las mujeres jóvenes. Algunas posibles explicaciones a esta dinámica pasan por el escaso emprendimiento femenino dentro de las TIC (Lamolla, 2010), o, de forma más determinante, por la elevada dificultad de conciliación familiar y laboral existente dentro del empleo TIC (Comisión Europea, 2018). En este sentido, existen teorías acerca del mayor grado de abandono que presentan las mujeres en el empleo TIC durante los años de tenencia de los hijos - drop-out phenomenon (Griffiths y Moore, 2010; Valenduc, 2011).

Si los gráficos anteriores muestran cuál es la evolución intragénero de la distribución ocupacional en el empleo TIC, un análisis complementario sería llevar a cabo la comparación inter-género. De este modo se podría observar en cada momento del tiempo y en relación con cada género cuál de las dos distribuciones queda más sesgada a la derecha o a la izquierda. Este análisis se consigue al calcular el Coeficiente de Asimetría de Fisher. Tal como se estableció previamente, su resultado determina cuál es la desviación con respecto a la media de la distribución de una variable. En nuestro caso, ha sido calculado para hombres y mujeres. Si el coeficiente toma valores positivos (negativos) se interpreta como un desplazamiento a la izquierda (derecha) de la distribución; es decir, existe un mayor sesgo del empleo TIC hacia las cohortes jóvenes (mayores) o de menor (mayor) edad. Si el coeficiente toma valor cero, la distribución sería completamente equilibrada o centrada.

La tabla II presenta los resultados obtenidos al calcular el Coeficiente de Asimetría de Fisher en el empleo TIC para hombres y mujeres. Los datos correspondientes a las mujeres presentan valores positivos para todos los años, excepto para 2014, y generalmente superiores a los datos relativos a los hombres. Ello establece que su distribución se encuentra más sesgada hacia las cohortes más jóvenes en relación con la distribución correspondiente a los hombres. Los valores promedio para ambos colectivos resumen dicha pauta. En el caso de los hombres la media del Coeficiente de Asimetría de Fisher para el periodo analizado es -0,0076 mientras que para las mujeres es 0,2670. Este hecho se fundamenta en que el empleo TIC ha sido tradicionalmente una ocupación de corte masculino lo cual provoca una escasa representación femenina en las cortes de edades más maduras. 
Tabla II: Coeficiente de Asimetría de Fisher para la distribución ocupacional de cada género en el empleo TIC, por cohortes de edad. Segundos trimestres $2011-2019$. (Fuente: EPA, INE).

\begin{tabular}{cccccccccc}
\hline & 2011 & 2012 & 2013 & 2014 & 2015 & 2016 & 2017 & 2018 & 2019 \\
\hline HOMBRES & 0,0173 & $-0,0459$ & $-0,1306$ & 0,1564 & $-0,0629$ & 0,1526 & 0,0888 & $-0,1113$ & $-0,1326$ \\
MUJERES & 0,3937 & 0,3760 & 0,0089 & $-0,0707$ & 0,5307 & 0,4729 & 0,1621 & 0,4396 & 0,0902 \\
\hline
\end{tabular}

Para finalizar este apartado y a modo de conclusión, es posible afirmar que el empleo TIC no es una ubicación laboral de elevada empleabilidad para los jóvenes, ni para el caso de los hombres ni para el de las mujeres. La distribución del empleo TIC para ambos géneros según la edad se desliza de forma lenta pero firme hacia cohortes maduras provocando un cierto "envejecimiento" de este tipo de empleo. Para los hombres, son importantes y preocupantes las reducciones porcentuales que se han producido en las cohortes de edad $25-29$ y $30-34$, mientras que en el caso de las mujeres la reducción más acusada se observa en las cohortes $30-34$ y $35-39$.

\subsection{Análisis econométrico de las cohortes en el empleo TIC}

Con el fin de confirmar los análisis previos, se ha desarrollado un modelo econométrico sobre la probabilidad de emplearse en una ocupación TIC frente al resto de ocupaciones ${ }^{9}$. Este modelo sirve de base para estimar las probabilidades predichas en la media para hombres y mujeres según su edad. Ello permite observar la posible empleabilidad laboral por género según la edad a partir de los determinantes laborales recogidos en el modelo. Adicionalmente, a partir del cálculo de dichas probabilidades es posible realizar una comparación temporal.

El modelo desarrollado se recoge en el anexo (tabla A.I) dado que nuestra atención se centra principalmente en los resultados obtenidos en relación a la edad. Esta estimación sigue el espíritu de investigaciones previas al incorporar los principales determinantes personales y laborales de la empleabilidad: ser mujer, tramos de edad en quinquenios,

\footnotetext{
9 Tal como se ha establecido previamente, el modelo seleccionado ha sido un "Probit" con sesgo de selección según Heckman (Heckman, 1979). Dicho modelo ha sido desarrollado en Stata bajo la orden "heckprobit".
} 
estar casado, ser padre o madre ${ }^{10}$, tener estudios secundarios, tener estudios superiores, trabajar a jornada completa, trabajar bajo un contrato indefinido y dummies relativas a las Comunidades Autónomas (Iglesias et al., 2010a) ${ }^{11}$.

De sus resultados se confirma el mantenimiento de algunas características claves en las TIC. Las mujeres presentan una menor probabilidad que los hombres de emplearse dentro de las TIC y dicha pauta se ha intensificado durante los últimos años. En concreto, si en el año 2015 las mujeres tenían un 4,7 por ciento menos de probabilidad de emplearse en una ocupación TIC frente al resto de ocupaciones, en el año 2019 este porcentaje ha aumentado a un 5,4 por ciento. Por otra parte, las TIC presentan un elevado grado de formación entre sus empleados. De manera concreta, es posible afirmar que el hecho de tener estudios superiores hace que una persona tenga el doble de probabilidad de trabajar en un empleo TIC frente a las personas con estudios secundarios

Los empleos TIC suelen ser ubicaciones laborales donde es más probable emplearse bajo contratos indefinidos, si bien en los últimos años ha aumentado la probabilidad de que dichos contratos tengan una duración menor a dos años. Es decir, aunque las TIC han sido un tipo de empleo con un alto grado de estabilidad laboral, también se puede establecer que la rotación laboral dentro de las ocupaciones TIC se ha visto incrementada.

El grafico 7 recoge las probabilidades predichas (calculadas en la media) de colocarse en una ocupación TIC frente al resto de ocupaciones para hombres y mujeres en el año 2015 y 2019 según la edad. Los resultados demuestran, por un lado, que las probabilidades estimadas para las mujeres resultan siempre inferiores a las probabilidades estimadas para los hombres independientemente de la edad considerada. En ambos colectivos la probabilidad de colocación en las TIC se reduce a medida que aumenta la edad de los trabajadores, si bien esta pauta resulta más acusada para el caso de las mujeres.

$\mathrm{Al}$ comparar cada género entre 2015 y 2019 , se puede establecer que los hombres han aumentado la probabilidad de estar empleados en las ocupaciones TIC principalmente en edades medias y maduras (a partir de los 40 años de edad). Esta tendencia no se ha producido entre

\footnotetext{
10 En la EPA no se pueden definir las relaciones familiares pero si la convivencia con hijos en el mismo hogar. Bajo esta premisa se ha construido la variable denominada "Padre/Madre" recogiendo a personas que conviven con hijos en el hogar.

11 Por cuestiones de representación y significatividad, las variables independientes incluidas en el modelo han sido establecidas, en términos generales, como dummies.
} 
las mujeres. Este resultado está en línea con las conclusiones obtenidas previamente en relación al gráfico 6 .

Gráfico 7: Probabilidad predicha (at means) de emplearse en una ocupación TIC por género y edad (24-64 años). (Fuente: EPA, INE).

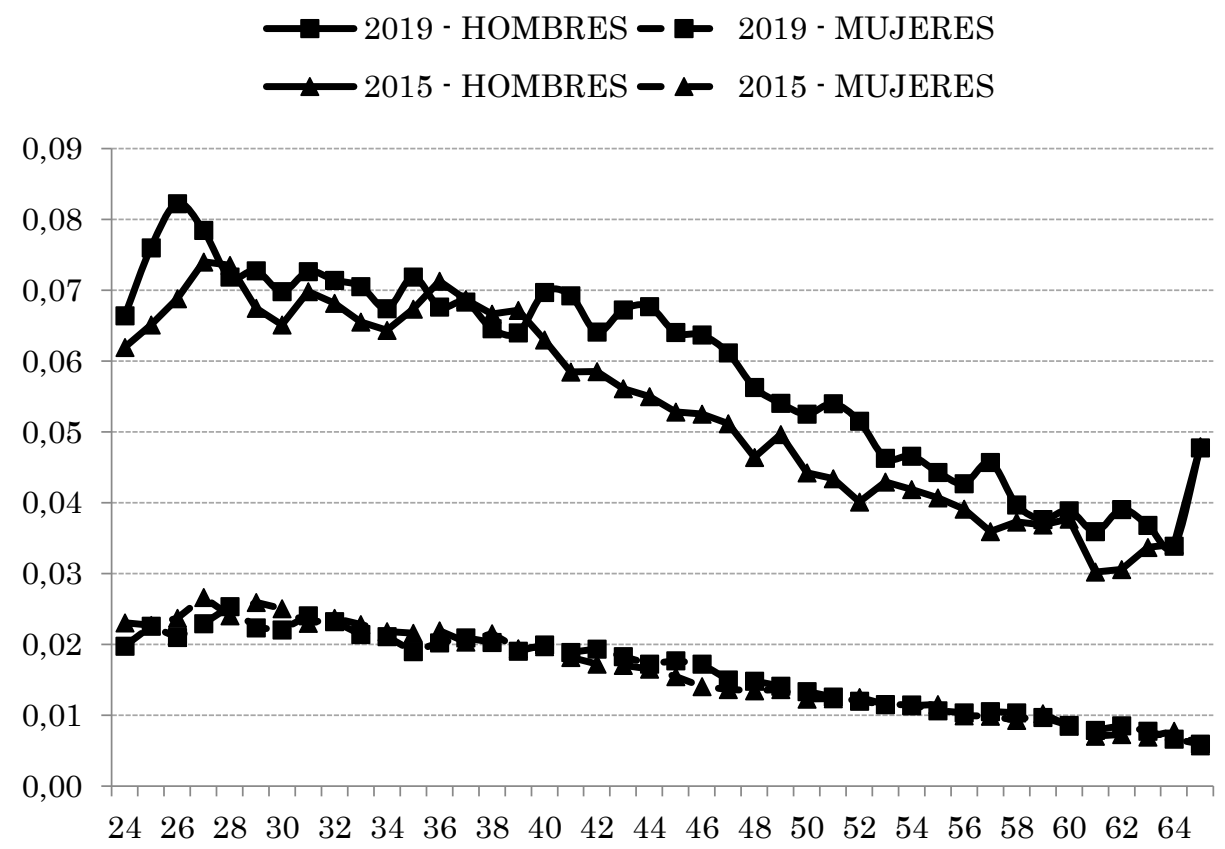

\subsection{La estabilidad laboral del empleo en las TIC}

En este apartado se pretende obtener una perspectiva sobre la estabilidad laboral existente dentro del empleo TIC tanto para hombres como para mujeres. Como aproximación se analiza la variable "Duración del contrato" 12 que recoge dentro de la EPA la información relativa a la duración del contrato actual sobre la base del número de meses que un trabajador acumula en la misma empresa y bajo un mismo puesto de trabajo. La información recogida en esta variable ha sido categorizada en 12 intervalos de 24 meses cada uno, a excepción del último tramo en el cual se aúna a aquellos trabajadores asalariados con una duración del contrato superior a 22 años. A partir de la distribución porcentual de dichos tramos es posible considerar que existe un mayor grado de

\footnotetext{
12 Referenciada en la metodología de la EPA como "DCOM"
} 
estabilidad cuando aumenta la presencia de los intervalos intermedios y superiores.

El gráfico 8 muestra los resultados obtenidos de este análisis para el conjunto de la población ocupada en empleo TIC en los segundos trimestres de los años 2011, 2015 y 2019. Los datos de este gráfico muestran la existencia de una distribución semejante a una campana de Gauss invertida. Los porcentajes más frecuentes se producen en la parte baja y alta de la distribución, que corresponden a los trabajadores con menor y mayor experiencia en la empresa. Por tanto, los datos indican que no se puede afirmar de forma rotunda que el empleo TIC se vincule directamente con la estabilidad laboral. Entre los años 2011 y 2019, la presencia de trabajadores con una menor experiencia (menos de 24 meses) se ha incrementado de manera continuada. Por consiguiente, el empleo TIC no es sinónimo de una mayor probabilidad de permanencia en la empresa.

En el gráfico 8 también se incluye un análisis similar para los hombres y las mujeres. En relación a los hombres, el 35,7 por ciento de ellos tenía una experiencia laboral en el mismo empleo TIC inferior a cuatro años para el año 2011. La misma ratio para el año 2019 ha aumentado hasta el 45,8 por ciento. En el extremo opuesto de la distribución, para el año 2011 , el 17,3 por ciento de los hombres en empleos TIC acumulaba una experiencia en la empresa entre 10 y 18 años. Sin embargo, este porcentaje aumentó únicamente al 17,7 por ciento en 2019.

En el caso de las mujeres se observan algunos datos más favorables. Tanto en 2011 como en 2019 el porcentaje de mujeres empleadas en las TIC con una experiencia en la empresa menor de cuatro años se sitúa en el 34 por ciento. Por otra parte, para las mujeres se observa un incremento del 21,8 por ciento al 23,5 por ciento en el tramo de experiencia laboral de 10 a 18 años. 
Gráfico 8: Distribución de la permanencia en el empleo TIC por meses. Total, hombres y mujeres. Segundos trimestres 2011, 2015 y 2019. (Fuente: EPA, INE).
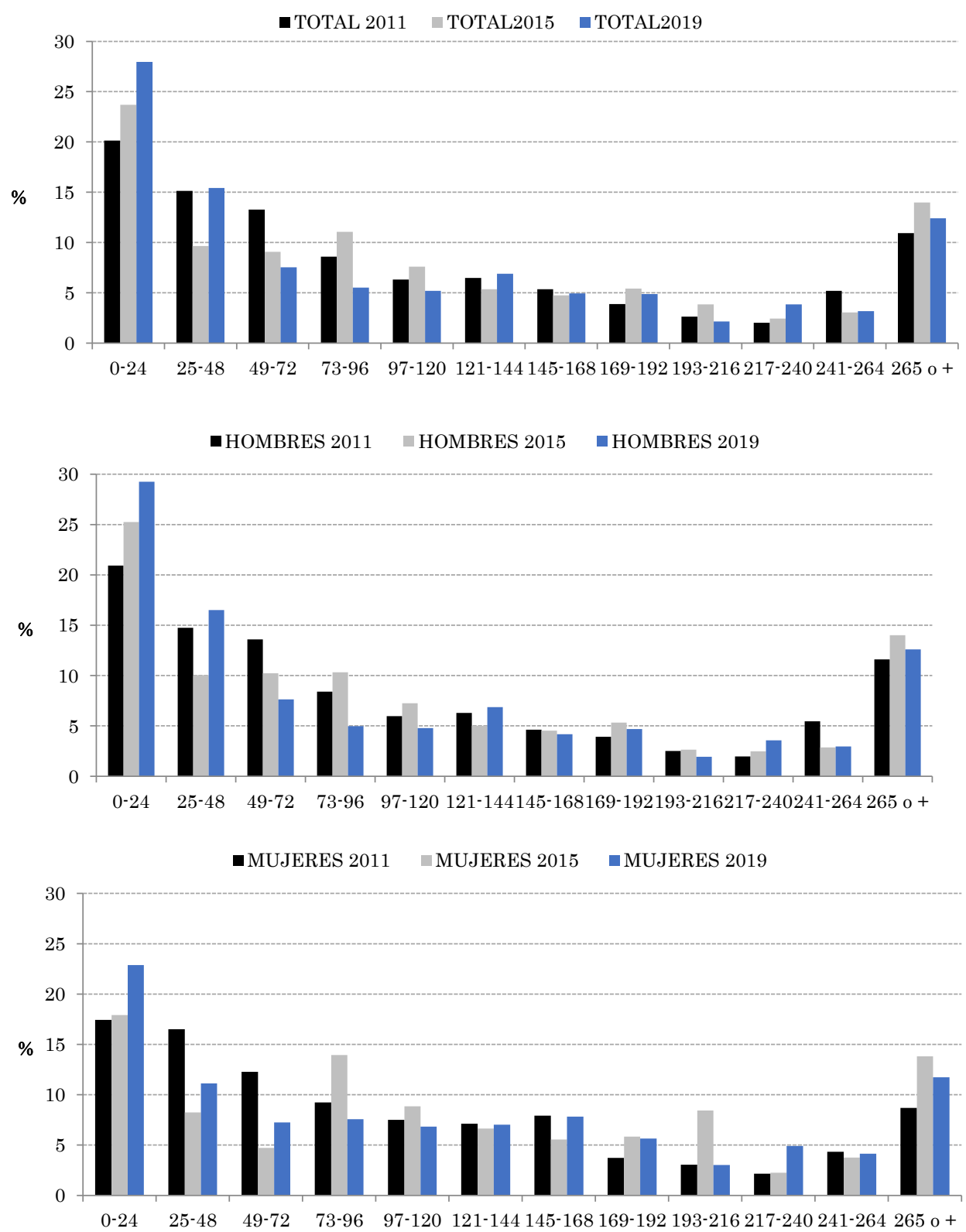
A modo de síntesis, el gráfico 9 recoge la tendencia exponencial que puede estimarse sobre las distribuciones previas relativas a los meses de permanencia en el empleo TIC de hombres y mujeres para el año 2011 y 2019. En los últimos nueve años, las mujeres han reducido su presencia en los tramos de menor experiencia o permanencia laboral para aumentarla en aquellos tramos de elevados niveles de experiencia laboral. No obstante, ambas variaciones presentan una intensidad muy reducida.

Por su parte, los hombres han reducido su presencia en todos los tramos de experiencia en la empresa según la tendencia estimada. No parece en definitiva que los empleos en el entorno de las TIC hayan mostrado en los últimos nueve años un perfil de estabilidad laboral para los hombres (entendiendo como tal la permanencia en la misma empresa). Si bien, también sería posible entender que estamos ante un tipo de empleo que podría haber aumentado su nivel de rotación laboral tras la crisis.

Gráfico 9: Estimación de la tendencia exponencial de la permanencia en la misma empresa para hombres (panel izquierdo) y mujeres (panel derecho) en empleos TIC por meses. Segundos trimestres 2011 y 2019. (Fuente: EPA, INE).
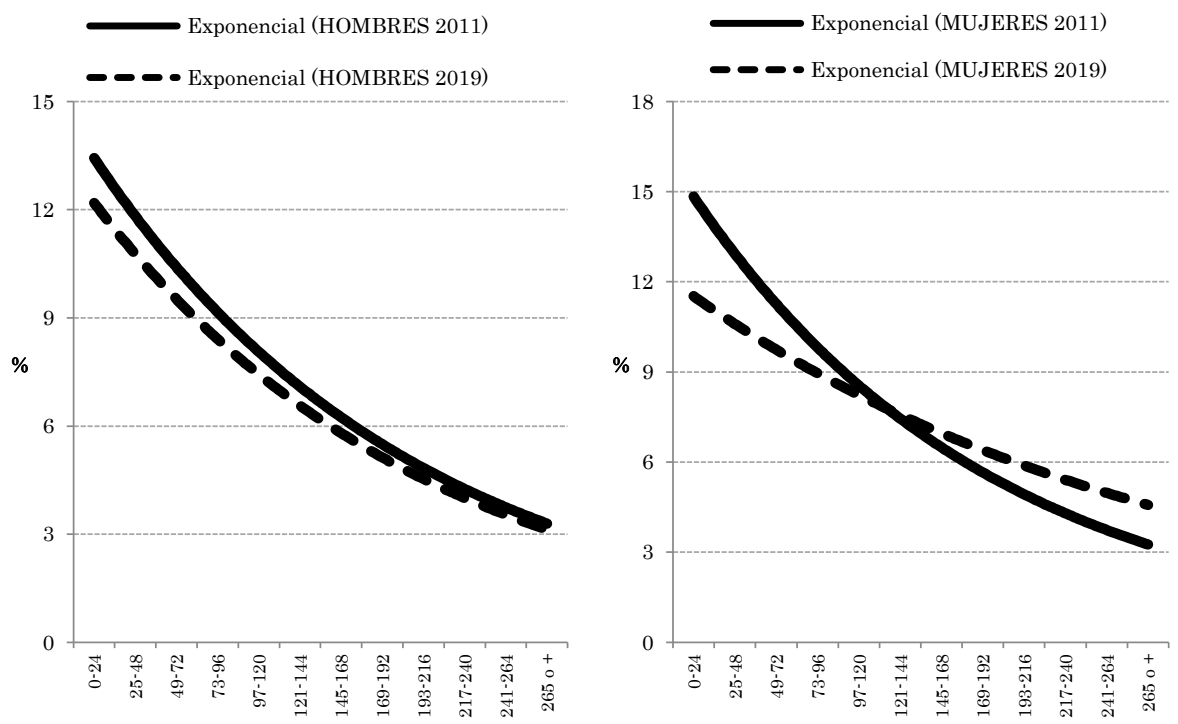


\section{Conclusiones}

Este artículo ha presentado una perspectiva actual de la relevancia del empleo TIC en España. Resulta necesario conocer cuál ha sido la evolución reciente de dicho tipo de empleo dadas las expectativas establecidas sobre su posible evolución. En primer lugar, se debe destacar el crecimiento que ha experimentado este tipo de empleo en los últimos años y su fuerte resistencia a la crisis económica siendo ciertamente un "refugio" laboral del empleo. Sin embargo, en segundo lugar, debe tenerse en cuenta que aún se trata de una ocupación laboral con una reducida presencia en el mercado de trabajo español. El empleo TIC muestra frente al resto de ocupaciones una mayor estabilidad laboral. Un par de variables laborales fundamentan esta afirmación: el mayor grado de contratación indefinida y el mayor desarrollo de una jornada laboral completa.

En términos de género es posible afirmar que es un tipo de empleo de carácter masculino ya que, aproximadamente, un 80 por ciento de los empleos están ocupados por hombres. Además, desde el año 2013 el número de hombres en las ocupaciones TIC ha experimentado un importante crecimiento (superior al 27 por ciento). Ello, afianza la hegemonía o mayor presencia de los hombres en este tipo de empleo.

Pese a esta masculinización, también se puede concluir que la segregación laboral es muy reducida con respecto a la que se evidencia en el resto de ocupaciones. La distribución interna del empleo resulta menos desigual respecto a otro tipo de ocupaciones, de manera que las TIC pueden ser un cierto referente en términos de igualdad ocupacional o distribución interna del empleo. Sería importante y necesario que las administraciones públicas fomentaran un mayor grado de acceso de las mujeres a este tipo de empleo.

El análisis de cohortes de edad ha posibilitado obtener algunos resultados de gran importancia. En primer lugar, el empleo TIC no es un destino laboral preferente para la incorporación masiva de los jóvenes. En este sentido, los resultados muestran un aumento de la edad media entre los trabajadores TIC. Además, existe un desplazamiento hacia la derecha de la distribución porcentual por tramos de edad tanto para el empleo TIC correspondiente a los hombres como a las mujeres. En segundo lugar, los resultados econométricos confirman la menor probabilidad de colocación de los jóvenes en el empleo TIC, estableciendo que dicha pauta adquiere mayor fuerza en el caso de los hombres. En tercer lugar, tampoco es posible afirmar de forma contundente que el 
empleo TIC sea sinónimo de estabilidad laboral en lo que se refiere a la permanencia en el mismo puesto de trabajo. Para los hombres este fenómeno se evidencia con más fuerza que para las mujeres; pero en ambos casos, se observa un cambio estructural marcado por la mayor presencia o peso de los trabajadores con menor tiempo de permanencia en el empleo TIC entre 2011 y 2019.

Con estos resultados, como corolario es posible afirmar que las ocupaciones TIC presentan un compendio de luces y sombras, si bien se demuestra que existe una falta de empleabilidad de la población más joven dentro del empleo TIC, independientemente de su género. En un mundo globalizado e interconectado por los avances de las tecnologías y de las comunicaciones, esta falta de empleabilidad de los jóvenes dentro de las TIC debería tenerse muy en cuenta en el desarrollo de las políticas de inserción laboral, con mayor motivo si se tiene en cuenta que los procesos educativos de estos jóvenes desde su infancia hasta la formación superior han sido vehiculados a través de las nuevas tecnologías.

\section{Referencias bibliográficas}

Arroyo, L. (2017). "La Digitalización y la Igualdad de Género en el Mercado de Trabajo", Revista de Estudios y Cultura, 81, 25-28.

Arroyo-Prieto, L. y Valenduc, G. (2016). "Digital Skills and Labour Opportunities for Low-Skilled Woman”, Working Papers Series, 6, Universidad de Hertfordshire.

Cabero, J., y Ruiz-Palmero, J. (2018). "Las Tecnologías de la información y la comunicación para la inclusión: reformulando la brecha digital". International Journal of Educational Research and Innovation, 9, 16-30

Carnoy, M. (2002). Sustaining the New Economy. Work, Family and Community in the Information Age. Cambridge, Massachusetts: Harvard University Press.

Caridad Sebastian, M., \& Ayuso García, M. D. (2011). "Situación de la brecha digital de género y medidas de inclusión en España". Investigación bibliotecológica, 25(55), 227-252.

Castaño, C. (2010). Género y TIC. Presencia, Posición y Políticas. Editorial UOC, Barcelona. 
Castaño, C. (2009). "Los usos de Internet en las edades más jóvenes: algunos datos y reflexiones sobre hogar, escuela, estudios y juegos. Participación educativa", 11. Revista cuatrimestral del Consejo Escolar del Estado: La educación, factor de igualdad, 73, 73-93.

Castaño, C. (dir.) (2008). La Segunda Brecha Digital. Editorial Cátedra, Madrid.

Castaño, C., Martín, J., y Martínez, J.L. (2011). "La Brecha Digital de Género en España y Europa: Medición con Indicadores Compuestos", Revista Española de Investigaciones Sociológicas, 136(1), 127-140.

Chen, D.H.C (2004). "Gender Equality and Economic Development. The Role for Information and Communication Technologies", World Bank Policy Research $W P, 3285,1-34$.

Collechia, A. y Papaconstantinou, G. (1996). "The Evolution of Skills in OECD Countries and the Role of Technology", Science, Technology, Industry (STI) WP, OECD, París, 1996/8, 1-37.

Comisión Europea (2018). Women in the Digital Age. DG Communications Networks, Content \& Technology. Luxemburgo.

Díaz-Chao, A. (2008). "Las Diferencias Salariales en la Economía del Conocimiento: un Análisis Empírico para España”, Revista sobre la Sociedad del Conocimiento, 6 , 1-7.

Ducatel, K y Burgelman, J.C. (1999). "The Futures Project Employment Map", Joint Research Centre, Bruselas: Comisión Europea, Series, número 3.

Dueñas, D., Iglesias, C. y Llorente, R. (2015). “Abordando la Desigualdad de Género. Empleo en Tecnologías de la Información y la Comunicación y Diferencias Salariales por Género en España”, Ensayos de Política Económica 33 (78), 207-219.

Duncan, O. y Duncan, J. (1955). "A Methodological Analysis of Segregation Indexes", American Sociological Review, 20, 210-217.

Fernández, V., Larraza, E., Ruiz, T. y Maritxalar, M. (2008). "Una Aproximación a la Situación de la Mujer en los Estudios Universitarios de Informática”, Arbor, 184 (733), 877-887.

Garrido, L. y Chuliá, E. (2005). Ocupación, Formación y el Futuro de la Jubilación en España. Consejo Económico y Social. 
Gil-Juarez, A., Vitores, A., Feliú, J. y Vall-Llovera, M. (2011). Brecha Digital de Género: una Revisión y una Propuesta, Teoría de la Educación. Educación y Cultura en la Sociedad de la Información, 12(2), 25-53.

Griffiths, M. y Moore, K. (2010). “'Disappearing Women”: A Study of Women Who Left the UK ICT Sector", Journal of Technology Management and Innovation, 5 (1), 95-107.

Heckman, (1979). "Sample Selection Bias as a Specification Error", Econometrica, n ${ }^{\circ} 47,153-161$.

Iglesias, C., Llorente, R. y Dueñas, D. (2010a). "Diferencias de Género en el Empleo TIC", Cuadernos de Economía, 33 (92), 105-138.

Iglesias, C., Llorente, R. y Dueñas, D. (2010b). "Desigualdad Salarial por Género en el Empleo de las Tecnologías de la Información y la Comunicación: El Caso Español”, Revista de Economía Laboral, 7, 125-151.

Iglesias, C., Llorente, R. y Dueñas, D. (2010c). "ICT Employment, Overeducation and Gender in Spain. Do Information and Communication Technologies Improve the Female Labour Situation?", New Technology, Work and Employment, 25 (3), 238-252.

Karmel, T. y MacLachlan, M. (1988). "Occupational Sex Segregation, Increasing or Decreasing?", The Economic Record, 64, 187-195.

Lamolla, L. (2010). "Las Mujeres Emprendedoras en el Sector TIC". En: Castaño, C., (coord..) Género y TIC: Presencia, Posición y Políticas. Editorial UOC.

Malo, M.A. y Cueto, B. (2014). "Young Employment in Spain: From the Blockade to the Youth Guarantee", Munich Personal RePEc Archive, 59473.

Martínez, J.L. y Castaño, C. (2017). "La Brecha Digital de Género y la Escasez de Mujeres en las Profesiones TIC", Panorama Social, (25), 49-65.

OCDE (2004). OECD Information Technology Outlook, 2004. OCDE, París.

OCDE (2014). Measuring the Digital Economy: A New Perspective. OECD Publishing.

OCDE (2017). "How Technology and Globalisation are Transforming the Labour Market”, Employment Outlook 2017, 81-124, OECD Publishing, París. 
Otobe, N. (2011). Global economic crisis, gender and employment: The impact and policy response(No. 994599383402676). International Labour Organization.

Palacio, J.I. y Simón, H. (2006) "Segregación Laboral y Diferencias Salariales por Razón de Sexo en España”, Estadística Española, 48 (163), 493-524.

Razavi, S. (2011). "World Development Report 2012: Gender equality and development: An opportunity both welcome and missed (an extended commentary)". United Nations Research Institute for Social Development.

Rendón, T. y Salas, C. (2000). Segregación y Diferencias Salariales por Género. Hipótesis y Evaluación Empírica, Informe final, Secretaría de Trabajo y Previsión Social. México.

Sanz, V. (2008). "Mujeres e Ingeniería Informática: el Caso de la Facultad de Informática de la UPM", Arbor, 184 (733), 905-915.

Segovia- Pérez, M., Castro Núñez, R. B., Santero Sánchez, R., \& Laguna Sánchez, P. (2019). "Being a woman in an ICT job: an analysis of the gender pay gap and discrimination in Spain". New Technology, Work and Employment. July.

Toharia, L., Moreno, G. y Cebrián, I. (1997). "Las Transiciones Laborales de las Mujeres Casadas en España, 1987-1996”, Información Comercial Española, 760, 129-143.

Valenduc, G. (2011). "Not a Job for Life? Women's Progression, Conversion, and Dropout in ICT Professions", International Journal of Gender, Science and Technology, 3 (2), 483-500.

WWW-ICT (2004). "Conceptual Framework and State of the Art. Widening Women's Work in Information and Communication Technology". www-ICT.com 
Anexo.

Tabla A.I. Probabilidad de emplearse en una ocupación TIC frente al resto de ocupaciones. Modelo Probit con sesgo de selección de Heckman. (Fuente: Elaboración propia sobre datos de la EPA, INE).

\begin{tabular}{|c|c|c|c|c|c|c|}
\hline & \multicolumn{3}{|c|}{2019} & \multicolumn{3}{|c|}{2015} \\
\hline & Coef. & $\mathrm{P}(\mathrm{Z}<\mathrm{z})$ & $\begin{array}{c}\text { Efecto } \\
\text { marginal: } \\
\text { dy/dx }\end{array}$ & Coef. & $\mathrm{P}(\mathrm{Z}<\mathrm{z})$ & $\begin{array}{c}\text { Efecto } \\
\text { marginal: } \\
\text { dy/dx }\end{array}$ \\
\hline Mujer & $-0,676$ & $0,000 * * *$ & $-0,054$ & $-0,597$ & $0,000 * * *$ & $-0,047$ \\
\hline Edad & $-0,011$ & $0000^{* * *}$ & $-0,001$ & $-0,011$ & $0,000 * * *$ & $-0,001$ \\
\hline Casado & 0,093 & $0,019^{* *}$ & 0,007 & 0,01 & 0,813 & 0,001 \\
\hline Padre/Madre & $-0,039$ & 0,38 & $-0,003$ & $-0,047$ & 0,323 & $-0,004$ \\
\hline Estudios secundarios & 0,814 & $0,001^{* * *}$ & 0,065 & 0,618 & $0,002^{* * *}$ & 0,049 \\
\hline Estudios superiores & 1,665 & $0,000^{* * *}$ & 0,134 & 1,337 & $0,000^{* * *}$ & 0,105 \\
\hline Jornada Completa & 0,474 & $0,000 * * *$ & 0,038 & 0,52 & $0,000 * * *$ & 0,041 \\
\hline Contrato indefinido & 0,331 & $0,000^{* * *}$ & 0,027 & 0,251 & $0,000^{* * *}$ & 0,020 \\
\hline $\begin{array}{l}\text { Duración del contrato (+ } \\
\text { de } 2 \text { años) }\end{array}$ & $-0,142$ & $0,000 * * *$ & $-0,011$ & $-0,104$ & $0,032^{* *}$ & $-0,008$ \\
\hline Constate & $-2,607$ & $0,000 * * *$ & & $-2,356$ & $0,000 * * *$ & 0,000 \\
\hline Dummies CCAA & SI & & & & & \\
\hline \multicolumn{7}{|c|}{ Ecuación de selección } \\
\hline Mujer & $-0,32$ & $0,000^{* * *}$ & & $-0,284$ & $0,000^{* * *}$ & \\
\hline Edad (35-44) & 0,101 & $0,000 * * *$ & & 0,057 & $0,002^{* * *}$ & \\
\hline Edad (45-54) & $-0,059$ & $0,002^{* * *}$ & & $-0,051$ & $0,006^{* * *}$ & \\
\hline Edad (55 y más) & $-1,156$ & $0,000 * * *$ & & $-1,085$ & $0,000^{* * *}$ & \\
\hline Casado & $-0,039$ & $0,002^{* * *}$ & & $-0,008$ & 0,561 & \\
\hline Padre/Madre & 0,352 & $0,000 * * *$ & & 0,297 & $0,000 * * *$ & \\
\hline Estudios secundarios & 0,781 & $0,000 * * *$ & & 0,734 & $0,000 * * *$ & \\
\hline Estudios superiores & 1,219 & $0,000 * * *$ & & 1,201 & $0,000^{* * *}$ & \\
\hline C. de Madrid & 0,223 & $0,000 * * *$ & & 0,231 & $0,000 * * *$ & \\
\hline C. Valenciana & 0,024 & 0,203 & & $-0,001$ & 0,959 & \\
\hline Cataluña & 0,179 & $0,000 * * *$ & & 0,223 & $0,000 * * *$ & \\
\hline Constate & $-0,385$ & $0,000 * * *$ & & $-0,513$ & $0,000^{* * *}$ & \\
\hline /athrho & $-0,182$ & $0,030^{* * *}$ & & $-0,185$ & $0,007^{* * *}$ & \\
\hline rho & $-0,180$ & & & $-0,183$ & & \\
\hline
\end{tabular}


Tabla A.I. Probabilidad de emplearse en una ocupación TIC frente al resto de ocupaciones. Modelo probit con sesgo de selección de Heckman. (Fuente: Elaboración propia sobre datos de la EPA, INE). Continuación.

\begin{tabular}{lcc}
\hline $\mathrm{N}^{\circ}$ de observaciones & 50145 & 45298 \\
\hline Wald chi2(25) & 842,99 & 476,57 \\
Log pseudolikelihood: & -1770000000 & -1750000000 \\
Prob > chi2 & $0,000^{* * *}$ & $0,000^{* * *}$ \\
\hline
\end{tabular}

Nota: Errores estándares - robustos.

La ecuación de selección resulta significativa.

*** Significatividad al $99 \%$ ** Significatividad al $95 \%$ y * Significativo al $90 \%$ 